\title{
Reducing Packet loss in Active Queue Management
}

\author{
Hariom Soni \\ M Tech. Scholar \\ Department of CSE, \\ OIST, Bhopal
}

\author{
Pradeep Kumar Mishra \\ Asst. Professor \\ Department of CSE, \\ OIST, Bhopal
}

\begin{abstract}
Random Early Detection (RED) is a network congestion control algorithm which detects incipient congestion according to current length of average queue. It detects congestion only after a packet has been dropped. In the recent, high speed networks, it is important to have some mechanisms that keep throughput high and average queue sizes low without packet loss and it would be undesirable to have large queue because it would significantly increase the average delay in the network.
\end{abstract}

\section{INTRODUCTION}

Mobile Ad hoc Network (MANET) [1] [2] is self-organizing network of mobile devices without any fixed infrastructure. Dynamic topology provides a great facility for ad hoc networks, Such as easy installation and mobility without loss of connection. In such facility packet dropping is a serious challenging for quality performance of ad hoc network. Dynamic nature of MANET with limited resources, which varies with time such as battery power, storage space, bandwidth constraint makes QoS provisioning, a challenging problem. Congestion within the network happens when the aggregated demands exceed available resources, and packet loss rate becomes serious drawback. In these increasing network resources is unable to address the congestion problem. Various congestion control methods have been proposed to solve this issue.

Packet loss rate is especially high during times of heavy congestion, when a large number of connections compete for limited network bandwidth. Due to an exponential increase in network traffic, many congestion control mechanisms have been proposed, including the deployment of explicit congestion notification (ECN) [4], along with active queue management (AQM) techniques. RED algorithm can solve the full queue problem by predicting the network congestion situation in advance. Before the queue size exceeds the capacity of the queue buffer, RED algorithm performs a precautionary measure to regulate the queue size in the queue buffer of the gateway. It drops the arriving packet occasionally and randomly before the full queue problem is encountered, so the problem of lock-out and global synchronization can be solved simultaneously [1]-[4].

RED detects congestion only after a packet has been dropped by router in the transmission. In the recent, high speed networks, it is important to have some mechanisms that keep throughput high but average queue sizes low without packet loss. To minimize packet loss it would be undesirable to have large queue because it would significantly increase the average delay in the network.

\section{RANDOM EARLY DETECTION}

Floyds et al proposed Random Early Detection (RED) [3] in 1993. The basic idea of this mechanism was to detect incipient congestion by monitoring the average queue length. Once the incipient congestion is detected, router selects the source terminal to notify about congestion. On reception of signal source terminal reduces the data transmission rate, to avoid queue overflow, and try to alleviate the network congestion. RED [4][5] algorithm consists of two steps: the first step is to calculate the average queue length, and the second step calculate the packet drop probability. Packet drop probability to decides whether to drop the packet or not, packet drop is treated as the signal of congestion.

\section{A. Calculation of the Average Queue Length} RED calculates the average queue length $\left(\operatorname{Avg}_{\mathrm{q}}\right)$, by using the following formula:

$$
\operatorname{Avg}_{\mathrm{q}}=\left(1-\mathrm{W}_{\mathrm{q}}\right) * \operatorname{Avg}_{\mathrm{q}}+\mathrm{q} * \mathrm{~W}_{\mathrm{q}}
$$

Here, $\mathrm{W}_{\mathrm{q}}$ represents the weighted value, and $\mathrm{q}$ represents the actual queue length in the sampling moments.

\section{B. Calculation of the Packets Drop Probability}

RED has two thresholds $\mathrm{Min}_{\text {th }}$ and $\mathrm{Max}_{\mathrm{th}}$, which are related with queue length. When the packet reaches the router, RED calculates the average of the queue length $\operatorname{Avg}_{\mathrm{q}}$ immediately. Then it determines the packet drop probability based on $\mathrm{Avg}_{\mathrm{q}}, \mathrm{Min}_{\text {th }}$ and $\operatorname{Max}_{\mathrm{th}}$. When $\operatorname{avg}_{\mathrm{q}}$ is greater than $\mathrm{Max}_{\mathrm{th}}$, all packets are discarded, and the packet loss rate is 1 . When $\operatorname{Avg}_{\mathrm{q}}$ is between $\mathrm{Min}_{\mathrm{th}}$ and $\mathrm{Max}_{\mathrm{th}}$, we have the following Packet Drop Probability (PDP) formula:

$$
\begin{aligned}
& \mathrm{P}_{\mathrm{b}}=\operatorname{Max}_{\mathrm{p}} *\left(\mathrm{Avg}_{\mathrm{q}}-\mathrm{Min}_{\mathrm{th}}\right) /\left(\mathrm{Max}_{\mathrm{th}}-\mathrm{Min}_{\mathrm{th}}\right) \ldots \ldots \\
& \mathrm{P}=\mathrm{P}_{\mathrm{b}} /\left(1-\text { count }^{*} \mathrm{P}_{\mathrm{b}}\right) \ldots \ldots \ldots \ldots \ldots \ldots \ldots \ldots \ldots \ldots \ldots \ldots \ldots \ldots \ldots \ldots \ldots \ldots
\end{aligned}
$$

Packet drop probability is used to decide whether to drop the packet or not, packet drop is treated as the signal of congestion.

\section{MODIFIED RANDOM EARLY DETECTION (MDRED)}

Proposed mechanism is based on virtually dividing the queue between minimum threshold $\left(\operatorname{Min}_{\mathrm{th}}\right)$ and maximum threshold $\left(\operatorname{Max}_{\mathrm{th}}\right)$ into smaller subparts and calculation of packet drop probability will be based on the position of average queue size (Avg) into smaller subparts. 


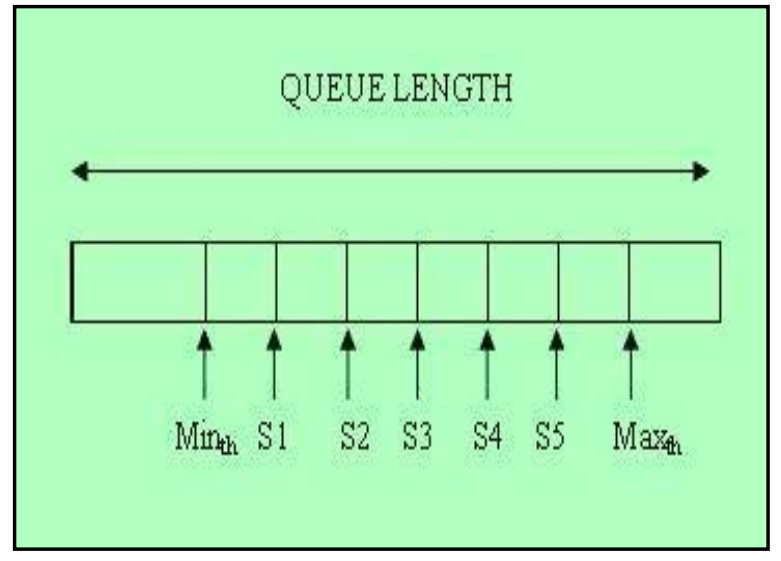

Figure 1: Queue length

Multiple values are applied between minimum threshold $\left(\mathrm{Min}_{\mathrm{th}}\right)$ and maximum threshold $\left(\mathrm{Max}_{\mathrm{th}}\right)$ in this case $\mathrm{S} 1, \mathrm{~S} 2, \mathrm{~S} 3, \mathrm{~S} 4, \mathrm{~S} 5$, five values are applied and it is divided into six smaller subparts.

\section{A. Calculation of the Average Queue Length}

MDRED calculates the average queue length (Avg), by using the following formula:

$\operatorname{Avg}=\left(1-\mathrm{W}_{\mathrm{q}}\right) * \operatorname{Avg}+\mathrm{q} * \mathrm{~W}_{\mathrm{q}}$

Here, $\mathrm{W}_{\mathrm{q}}$ represents the weighted value, and $\mathrm{q}$ represents the actual queue length in the sampling moments.

\section{B. Calculation of the Packets Drop Probability}

MDRED has two thresholds $\operatorname{Min}_{\text {th }}$ and $\mathrm{Max}_{\text {th }}$ and other value S1, S2, S3, S4, S5 which are related with queue length. When the packet reaches the router, it calculates the average of the queue length Avg immediately. Then it determines the packet drop probability based on Avg, Min ${ }_{\text {th }}$ and $\mathrm{Max}_{\mathrm{th}}$ and S1, S2, S3, S4, S5 .

If average queue size lies between minimum threshold and maximum threshold then Calculate Packet Drop Probability

If $\left(\operatorname{Min}_{\text {th }}<\operatorname{Avg}<\mathrm{S} 1\right)\{$

$\left.\mathrm{P}_{\mathrm{b}}=\operatorname{Max}_{\mathrm{p}} *\left(\operatorname{Avg}_{\mathrm{q}}-\operatorname{Min}_{\mathrm{th}}\right) /\left(\operatorname{Max}_{\mathrm{th}}-\operatorname{Min}_{\mathrm{th}}\right)\right\}$

Else if $(\mathrm{S} 1<=\operatorname{Avg}<\mathrm{S} 2)\{$

$\left.\mathrm{P}_{\mathrm{b}}=\operatorname{Max}_{\mathrm{p}} *\left(\operatorname{Avg}_{\mathrm{q}}-\mathrm{S} 1\right) /\left(\operatorname{Max}_{\mathrm{th}}-\mathrm{Min}_{\mathrm{th}}\right)\right\}$

Else if $(\mathrm{S} 2<=\mathrm{Avg}<\mathrm{S} 3)\{$

$\left.\mathrm{P}_{\mathrm{b}}=\operatorname{Max}_{\mathrm{p}} *\left(\operatorname{Avg}_{\mathrm{q}}-\mathrm{S} 2\right) /\left(\operatorname{Max}_{\mathrm{th}}-\mathrm{Min}_{\mathrm{th}}\right)\right\}$

Else if $(\mathrm{S} 3<=\mathrm{Avg}<\mathrm{S} 4)\{$

$\left.\mathrm{P}_{\mathrm{b}}=\operatorname{Max}_{\mathrm{p}} *\left(\operatorname{Avg}_{\mathrm{q}}-\mathrm{S} 3\right) /\left(\operatorname{Max}_{\mathrm{th}}-\mathrm{Min}_{\mathrm{th}}\right)\right\}$

Else if $(\mathrm{S} 4<=$ Avg $<$ S5 $)\{$

$\left.\mathrm{P}_{\mathrm{b}}=\operatorname{Max}_{\mathrm{p}} *\left(\operatorname{Avg}_{\mathrm{q}}-\mathrm{S} 4\right) /\left(\operatorname{Max}_{\mathrm{th}}-\operatorname{Min}_{\mathrm{th}}\right)\right\}$

Else if $\left(\mathrm{S} 5<=\mathrm{Avg}<\mathrm{Max}_{\mathrm{th}}\right)\{$
$\left.\mathrm{P}_{\mathrm{b}}=\operatorname{Max}_{\mathrm{p}} *\left(\operatorname{Avg}_{\mathrm{q}}-\mathrm{S} 5\right) /\left(\operatorname{Max}_{\mathrm{th}}-\mathrm{Min}_{\mathrm{th}}\right)\right\}$

$\mathrm{P}=\mathrm{P}_{\mathrm{b}} /\left(1-\right.$ count $\left.^{*} \mathrm{P}_{\mathrm{b}}\right)$

\}

If average queue size is greater than Maximum threshold $\left(\operatorname{Max}_{\mathrm{th}}\right)$ then

If $\left(\operatorname{Max}_{\mathrm{th}}<\right.$ Queue_length $)\{$

$\operatorname{Max}_{\mathrm{th}}=\operatorname{Max}_{\mathrm{th}}+$ packet_size

$\left.\mathrm{P}=\operatorname{Max}_{\mathrm{p}}\right\}$

Packet drop probability is used to decide whether to drop the packet or not, packet drop is treated as the signal of congestion.

\section{SIMULATION AND RESULTS}

\section{A. Throughput}

Throughput is the average rate of successful data delivery over a communication channel.

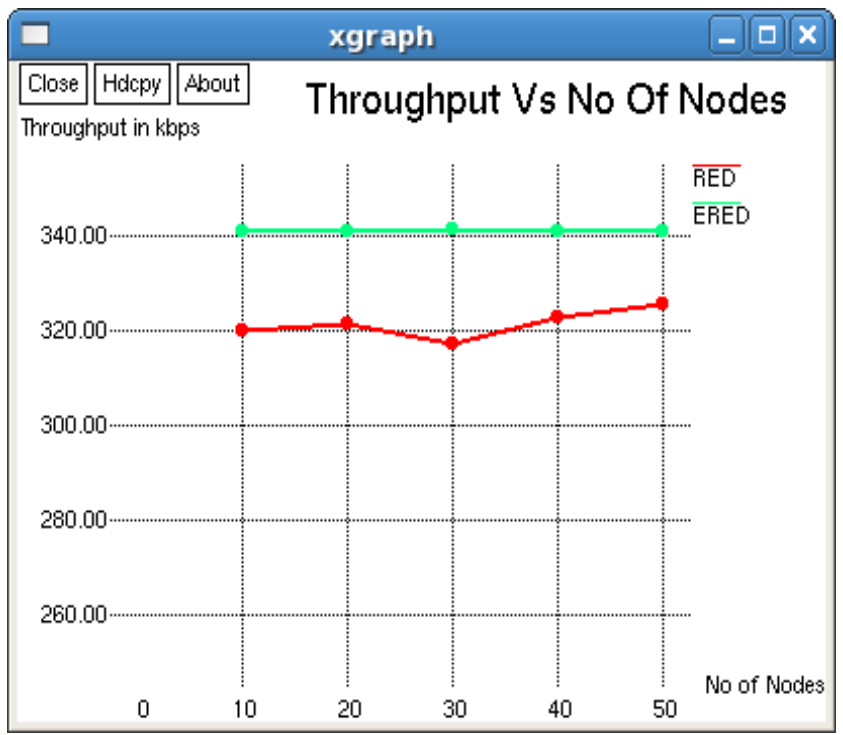

Figure 2: Throughput comparison xgraph 
Table 1: Throughput comparison table

\begin{tabular}{|c|l|l|}
\hline $\begin{array}{c}\text { No. of } \\
\text { nodes }\end{array}$ & RED & MDRED \\
\hline 10 & 320.03 & 341.07 \\
\hline 20 & 321.5 & 340.99 \\
\hline 30 & 317.27 & 341.60 \\
\hline 40 & 322.77 & 341.0 \\
\hline 50 & 325.62 & 340.9 \\
\hline
\end{tabular}

\section{B. Packet Delivery Ratio}

Packet Delivery Ratio is calculated as total delivered packet to the total no of transmitted packets.

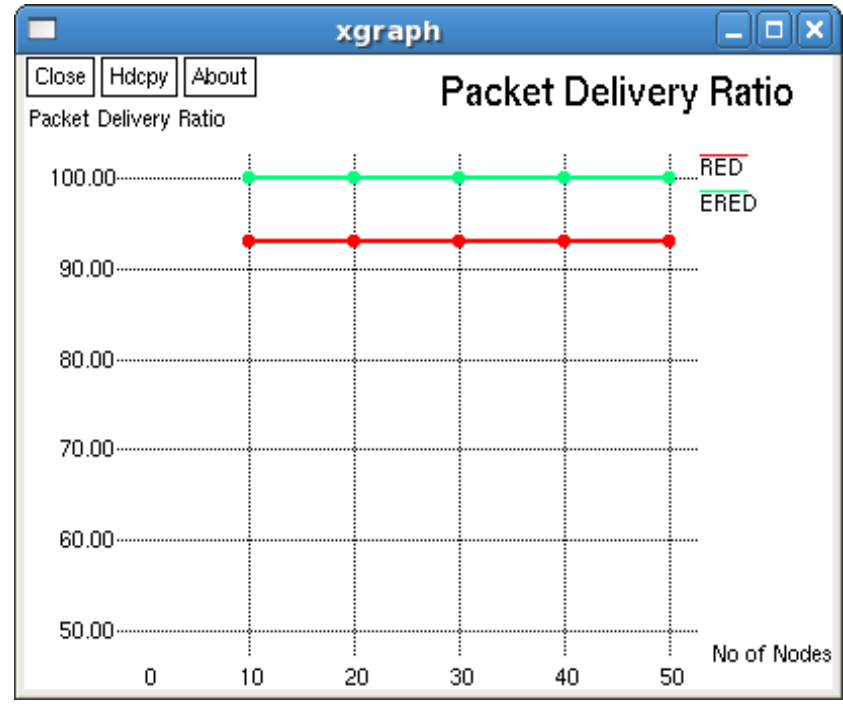

Figure 3: Packet Delivery Ratio comparison xgraph

From the figure 3 , it is confirmed that both line of packet delivery ratio is straight lines which shows that packet delivery ratio is consistent in each case of using different no of nodes configuration from 10 nodes to 50 nodes. From figure 3 it is shown that Random Early Detection, having Packet Delivery Ratio (PDR) consistent around 92 and proposed mechanism having PDR consistent around 100.

\section{Comparison of Packet transmitted v/s Packet received}

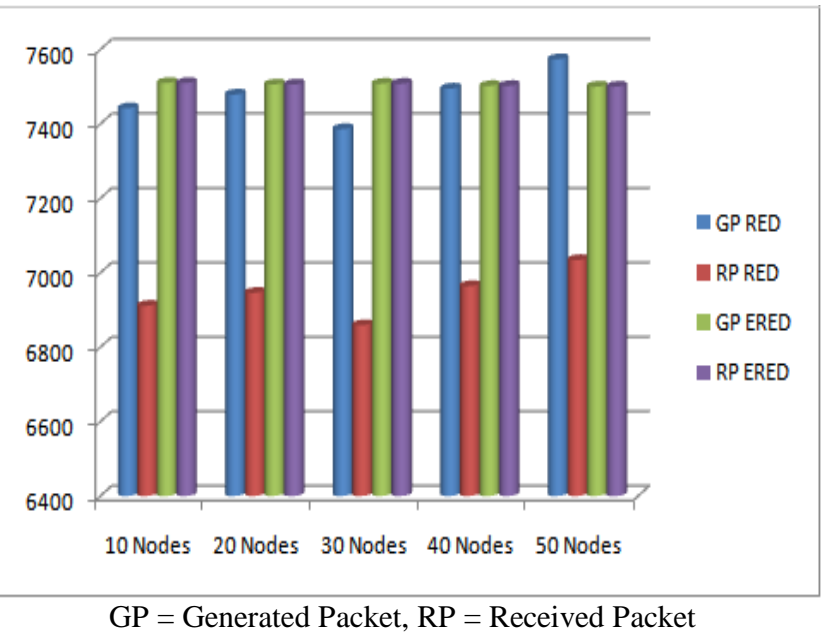

Figure 4: Packet transmitted v/s packet delivered comparison graph

Figure 4 having the comparison of both the Random Early Detection (RED) and proposed mechanism in term of packet transmitted and packet delivered at the destination. This graph shows that RED having the lots of packet drop thus there difference in packet transmitted and packet delivered, but in proposed mechanism there is no difference in packet transmitted and packet received thus there is no packet drop.

\section{CONCLUSION}

This paper proposed a new queue management algorithm based on a completely different control methodology. Without having to rely on some assumptions, new methodology allows the system to completely previous adjustment so as to decide on the trend of new adjustment. Compared to the standard RED, this proposed technique clearly demonstrates its superior performance. It is very likely the same methodology can be easily applied to the adjustment of other critical parameters in the system, for example, the $\max _{\mathrm{th}}$ and/or $\min _{\mathrm{th}}$. Packet Delivery Ratio and Throughput are employed as the performance indicator for merit judgment of the proposed technique.

\section{REFERENCES}

[1] Shensheng Tang and Wei Li. "QoS Provisioning and Queue Management in Mobile Ad hoc Networks", WCNC 2006: 14244-0270-0/06. 2006 IEEE.

[2] Vahid Ayatollahi Tafti and Abolfazl Gandomi. "Performance of QoS Parameters in MANET Application Traffics in Large Scale Scenarios" World Academy of Science, Engineering and Technology, 2010

[3] S. Floyd and V. Jacobson. "Random early detection gateways for congestion avoidance", IEEE/ACM Transactions on Networking, 1(4):397-413, 1993.

[4] V. Firoiu and M. Borden, "A study of active queue management for congestion control", In Proceedings of the IEEE Infocom, pages 1435-1444, Tel Aviv, Mar 2000.

[5] T. Alemu, Y. Calas, and A. Jean-Marie, "The Interaction of Forward Error Correction and Active Queue Management" 
Proceedings of the IFIP Networking'05, Waterloo, Ontario, Canada, 2005.

[6] Chin-Hui Chien; Wanjiun Liao,"A self-configuring RED gateway for quality of service (QoS) networks, Multimedia and Expo", 2003. ICME '03, Page(s): I - 793-6.

[7] A. Kuzmanovic, A. Mondal, S. Floyd, K. Ramakrishnan. RFC 5562 -"Adding Explicit Congestion Notification Capability to TCP's SYN/ACK Packets",AT\&T Labs Research, June 2009.

[8] K.Dinesh Kumar, I.Ramya \& M.Roberts Masillamani, "Queue Management in Mobile Adhoc Networks (Manets)" 2010 IEEE.
[9] Zhenyu $\mathrm{Na}$ and Qing Guo "An Improved AQM Scheme with Adaptive Reference Queue Threshold" 978-1-4577, 2011 IEEE.

[10] Guan-Yi Su and Chian C. Ho "Random Early Detection Improved by Progressive Adjustment Method" Proceedings of IEEE 2008 6th National Conference on Telecommunication Technologies and IEEE 2008 2nd Malaysia Conference on Photonics, August 2008, Putrajaya, Malaysia. 2008 IEEE.

[11] Rob Torres, John Border, George Choquette, Jun Xu, and Je-Hong Jong, "Congestion Control using RED and TCP Window Adjustment” 978-1-4673, 2013 IEE. 